\title{
Passive range of motion changes in young children with spastic diplegia. A study during the initial stages of independent walking
}

\author{
Rigas Dimakopoulos ${ }^{\mathrm{a}, *}$, George Syrogiannopoulos ${ }^{\mathrm{a}}$, Sotirios Youroukos $^{\mathrm{b}}$, Zoe Dailiana ${ }^{\mathrm{c}}$ and \\ Arietta Spinou $^{\mathrm{d}}$ \\ ${ }^{a}$ Department of Pediatrics, Faculty of Medicine, University of Thessaly, Larissa, Greece \\ ${ }^{\mathrm{b}}$ First Pediatric Clinic, University of Athens, "Agia Sofia" Children's Hospital, Athens, Greece \\ ${ }^{\mathrm{c}}$ Departments of Orthopaedic Surgery, Faculty of Medicine, University of Thessaly, Larissa, Greece \\ ${ }^{\mathrm{d}}$ School of Health Sport and Bioscience, University of East London, London, UK
}

\begin{abstract}
.
PURPOSE: To investigate how the onset of independent walking in children with Cerebral Palsy (CP) influences the Passive Range of Motion (PROM) of lower limb joints.

METHOD: Sixteen children with CP, GMFCS levels I and II, and 16 Typical Development (TD) children who had just begun independent walking participated in the study. The PROM of the hip abduction and external rotation, knee extension, popliteal angle, and ankle dorsiflexion was recorded with a goniometer at the onset of independent walking and 8 months later. A repeatedmeasures two-way ANOVA was conducted to compare the main effects of "walking experience" and "group of children" and the interaction effect between them on PROM of lower extremities' joints.

RESULTS: The effect of "group of children" in the PROM was significant; differences were observed between children with $\mathrm{CP}$ and TD children for all joints $(p<0.05)$. The effect of "walking experience" did not have a significant impact on PROM changes and the interaction effect of "group of children" and "walking experience" was also not significant for all the variables. CONCLUSIONS: The alternative gait pattern spontaneously adopted by children with CP does not significantly impact their PROM during the initial stages of walking development. The lower PROM in highly functional children with CP compared to TD children cannot be attributed to gait initiation with an "atypical" pattern, but possibly to other factors such as reduced voluntary movement and age.
\end{abstract}

Keywords: Passive range of motion, cerebral palsy, gait, goniometry, spasticity

\section{Introduction}

Cerebral Palsy (CP) represents the most common cause of motor disability in children, with an incidence of 2-2.5 per 1,000 births [1]. This condition by definition affects movement and posture, and therefore walking [2]. Studies indicate that $30.6 \%$ of all children with

${ }^{*}$ Corresponding author: Rigas Dimakopoulos, Deliyanni 1 and Themistokleous 18, 151 22, Maroussi, Athens, Greece. Tel.: +30 6977259 939; E-mail: rigasd@yahoo.com.
CP will not be able to walk independently (Gross Motor Function Classification System, [GMFCS], levels IV and V), $11.3 \%$ will walk independently using a mobility device (GMFCS III), while $58.2 \%$ will be able to walk independently though with certain restrictions (GMFCS I and II) [3,4].

A parameter associated with the development of gait in children with CP is the lower limb joints' Passive Range of Motion (PROM), while its restriction often relates to musculoskeletal deformities. Indeed, most children with abnormally high muscle tone (spastic types of CP) display decreased PROM and muscu- 
loskeletal deformities at some stage of their development [5], which usually worsens as age increases [6,7]. This is important, as in many cases the treatment of the restricted PROM by means of botulinum toxin, orthotics, and orthopedic interventions contributes significantly in enhancing the movement and quality of life of children with CP [8].

On the other hand, Typical Development (TD) children, although they do not present any abnormality in gait pattern or muscle tone, also demonstrate decreasing values in the PROM of their lower limbs' joints as age increases. The reference values for decreasing PROM vary according to the joint, but for some joints of the lower extremities they reach up to $-1^{\circ}$ per year $[9,10]$. Thus, the general tendency in children is a progressive restriction in PROM with increasing age.

Currently, many clinicians follow traditional therapeutic methods based on the normalization of muscle tone and movement. Under these concepts, "atypical" movement patterns often adopted by children with $\mathrm{CP}$ are not encouraged because they are thought to contribute to the establishment of pathological movement patterns, restriction of PROM, and consequently to the development of musculoskeletal deformities [11,12]. In contrast, more recent treatment concepts stress autonomy, functionality, and encourage the use of "alternative" movement methods if they improve independence and movement efficiency [13]. Understanding whether or not "atypical" gait patterns lead to greater PROM limitations is of utmost interest and could have significant clinical implications.

Although the decrease in the PROM is recognised as a major restricting factor in the overall motor skills and functionality in children with $\mathrm{CP}$, the factors that influence it are not currently fully understood [14]. In addition, the relationship between gait initiation and changes in PROM is unclear, considering that only few studies investigate the PROM for children with CP under 4 years of age [15]. As a result, there is a paucity of data with regards to the PROM of the lower limbs for children with CP who are still in the initial stages of walking development, and about how their PROM compares to TD children.

The aim of this study was to investigate the changes in the PROM of the lower limb joints in young children with CP, GMFCS level I and level II, during the initial stages of gait development. We hypothesised that significant changes in PROM observed during the first 8 months of gait development should indicate that the onset of independent walking has an important impact on PROM progression. If such a hypothesis was supported, it could provide a preliminary indication whether the onset of independent walking with an "atypical" gait pattern by children with CP might contribute to the development of future musculoskeletal deformities. We also aimed to compare the PROM of children with $\mathrm{CP}$ and TD children with similar walking experience in terms of walking duration. We hypothesized that children with $\mathrm{CP}$ display lower values of PROM compared to TD children, independently of their walking phase.

\section{Methods}

\subsection{Participants}

Children with $\mathrm{CP}$ and a control group of healthy TD children were recruited. Inclusion criteria for the children with CP were: diagnosis of $\mathrm{CP}$, spastic diplegia subtype based on magnetic resonance imaging and relevant clinical presentation, and GMFCS level I and II [16]. All participants were $<4$ years old, had started walking independently within the past 2 months, and were able to walk a minimum distance of 5 meters without falling. For the purposes of this study, the age of walking onset was parent-reported and defined as the time in which the child was able to take at least 5 consecutive steps without falling. The study excluded children who displayed severe mental retardation, had changes in their clinical treatment during the study, had orthopaedic interventions that could have influenced the PROM of their lower limbs or were scheduled to undergo such an intervention in the next 8 months, had previously received an injection of botulinum toxin or were scheduled to receive one in the next 8 months, and children who had been previously administered muscle relaxant drugs in any form. TD children participated as a control group to provide data on how the PROM of lower limbs changes for the same walking period. This data would also indicate clinical significance for potentially observed PROM changes in children with CP.

\subsection{Protocol}

Children were recruited consecutively from the $1^{\text {st }}$ Pediatric Clinic of Agia Sofia Children's Hospital in Athens between December 2012 and July 2015. All measurements were conducted under the same conditions at the Gait Analysis Laboratory of the Greek Society for the Protection and Rehabilitation of Disabled 
Table 1

Demographics of the study participants $(n=32)$

\begin{tabular}{|c|c|c|c|c|}
\hline & \multicolumn{2}{|c|}{ Baseline } & \multicolumn{2}{|c|}{ Follow up } \\
\hline & $\begin{array}{c}\mathrm{CP} \\
(n=16)\end{array}$ & $\begin{array}{c}\text { TD } \\
(n=16)\end{array}$ & $\begin{array}{c}\mathrm{CP} \\
(n=16)\end{array}$ & $\begin{array}{c}\text { TD } \\
(n=16)\end{array}$ \\
\hline Sex (male) & 9 & 10 & 9 & 10 \\
\hline Age (months) & $28.9 \pm 8.7$ & $13.4 \pm 2.2$ & $36.9 \pm 8.7$ & $21.4 \pm 2.2$ \\
\hline Weight (Kg) & $11.0 \pm 1.2$ & $10.4 \pm 0.7$ & $12.6 \pm 1.5$ & $11.1 \pm 0.9$ \\
\hline Height $(\mathrm{cm})$ & $857 \pm 44.2$ & $795 \pm 23.1$ & $921 \pm 40.2$ & $887 \pm 36.5$ \\
\hline Lower limb length $(\mathrm{cm})$ & $398 \pm 32.7$ & $354 \pm 16.9$ & $435 \pm 32.0$ & $405 \pm 23.6$ \\
\hline PT frequency (sessions/week) & $1.93 \pm 0.7$ & - & $1.87 \pm 0.5$ & - \\
\hline OT frequency (sessions/week) & $0.6 \pm 0.7$ & - & $0.7 \pm 0.6$ & - \\
\hline
\end{tabular}

Data presented as means \pm standard deviation. CP: cerebral palsy, TD: typical development, GM-

FCS: gross motor function classification system; PT: physical therapy, OT: occupational therapy.

Persons (ELEPAP) of Athens. Baseline measurements were conducted within 4-8 weeks of the child's newlystarted independent walking and the follow-up after 8 months.

Usual care was followed throughout the study participation. All children with CP wore Ankle Foot Orthoses (AFOs) for 6 hours during sleep, while no AFOs were used during walking. Each child received weekly physical and occupational therapy sessions (Table 1) that were based on the principle of normalization of motor performance and quality of movement, as described by Ketelaar et al. [13].

Ethical approval for this study was provided by the scientific committee of the Medical School of the University of Thessaly and the Greek Committee of Ethics and Deontology of the University Hospital of Larissa. All carers of the participants provided informed consent according to the committee's requirements.

\subsection{Passive range of motion}

PROM of the joints of the hip, knee, and ankle of both lower limbs were assessed in all children using a standard goniometer and under standardised conditions. Goniometry is the most frequently used clinical evaluation method of the joints' PROM in children with CP. Its accuracy and reliability have been extensively studied and it has become an established method for evaluating PROM, with high inter-rater and intra-rater reliability [17-19]. Additionally, this study assessed inter-rater reliability of the goniometry, as all measurements were repeated independently by two specialist paediatric physiotherapists with $>10$ years of clinical experience.

The lower limbs movements and directions of evaluation were hip abduction, hip external rotation, knee extension, and ankle dorsiflexion. Those were selected based on the frequency with which they appear re- stricted (decreased PROM) in children with CP [17]. All measurements were performed with the child in the supine position, except for the measurement of hip rotation, which was conducted in the prone position. The sequence of recording was dorsiflexion of the ankle with the knee in full extension, extension of the knee with the hip in neutral position, popliteal angle with $90^{\circ}$ hip flexion, abduction of the hip with the knee in full extension, and external rotation of the hip with $90^{\circ}$ knee flexion.

\subsection{Statistical analysis}

IBM $^{\circledR}$ SPSS $^{\circledR}$ Statistics 19 was used for statistical analysis. Kolmogorov-Smirnov test was used to assess normality of distribution. A repeated-measures twoway analysis of variance (ANOVA) was conducted to compare the main effects of 2 variables, "group of children" and "walking experience", and the interaction effect between them on the PROM of lower extremities' joints. "Group of children" consisted of 2 levels (children with CP, TD children) and "walking experience" consisted also of 2 levels (beginning of walking$1^{\text {st }}$ measurement, 8 months of walking experience- $2^{\text {nd }}$ measurement). Ad hoc t-tests were used for the independent samples of children with CP and TD children that were found significant in the ANOVA. Inter-rater reliability of PROM measurements was analysed using the IntraClass Correlation Coefficient (ICC). Significance level was set at $\alpha=0.05$.

\section{Results}

Sixteen children ( 9 boys) with CP-Spastic Diplegia and 16 TD children (10 boys) were recruited. Participant demographics are presented in Table 1. Twelve children with CP were GMFCS level I and four were 

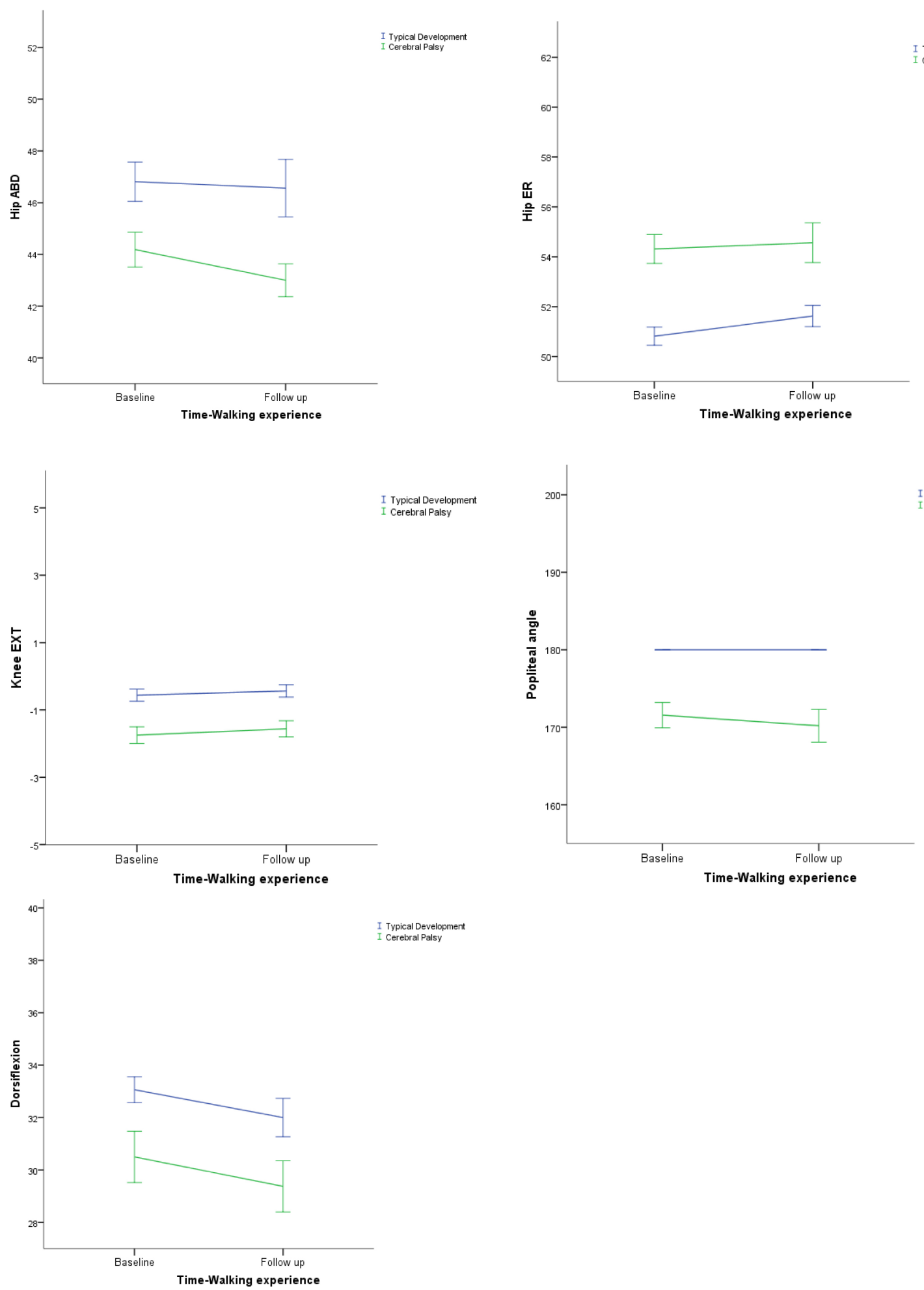

Fig. 1. Main effects of "walking experience" and "group of children" and the interaction effect between them on the PROM of hip abduction, hip external rotation, knee extension, popliteal angle, and dorsiflexion of the ankle. Data presented as means. Error bars represent \pm 1 standard error of the mean. ABD: abduction, ER: external rotation, EXT: extension. 
Table 2

Effect of time (walking experience), group (group of children) and their interaction in the passive range of motion (PROM) of the lower limb joints in children with cerebral palsy and typical development children

\begin{tabular}{|c|c|c|c|c|c|c|c|c|c|c|}
\hline & \multicolumn{2}{|c|}{ Baseline } & \multicolumn{2}{|c|}{ Follow up } & \multicolumn{2}{|c|}{ Time } & \multicolumn{2}{|c|}{ Group } & \multicolumn{2}{|c|}{ Time $*$ Group } \\
\hline & TD & $\mathrm{CP}$ & TD & $\mathrm{CP}$ & $\mathrm{F}(1,30)$ & $\mathrm{p}$ & $\mathrm{F}(1,30)$ & $\mathrm{p}$ & $\mathrm{F}(1,30)$ & $\mathrm{p}$ \\
\hline Hip ABD & 46.8 & 44.2 & 46.6 & 43.0 & 1.24 & 0.274 & 10.42 & 0.003 & 0.53 & 0.473 \\
\hline Hip ER & 50.8 & 54.3 & 51.6 & 54.6 & 1.21 & 0.280 & 25.14 & $<0.001$ & 0.34 & 0.564 \\
\hline Knee EXT & 0.6 & 1.7 & 0.4 & 1.6 & 5.60 & 0.250 & 15.00 & 0.001 & 0.22 & 0.640 \\
\hline Popliteal angle & 180.0 & 171.6 & 180.0 & 170.2 & 1.62 & 0.213 & 25.35 & $<0.001$ & 1.62 & 0.213 \\
\hline Ankle dorsiflexion & 33.0 & 30.5 & 32.0 & 29.4 & 3.17 & 0.850 & 6.94 & 0.013 & 0.03 & 0.960 \\
\hline
\end{tabular}

Data presented as means; $2 \times 2$ ANOVA, significance set at $p<0.05$. B: baseline, FU: follow-up, TD: typical development, CP: cerebral palsy,

ABD: abduction, ER: external rotation, EXT: extension.

level II. Figure 1 shows the PROM for the hip abduction and external rotation, knee extension and popliteal angle, and ankle dorsiflexion for children with $\mathrm{CP}$ and for those with TD, at baseline and follow-up.

All effects for the influence of "group of children" on the changes of PROM of lower extremities' joints were statistically significant, whilst for the "walking experience" factor and the interaction between "walking experience" and "group of children" were not (Table 2). Further ad hoc examination showed that PROM was decreased in children with $\mathrm{CP}$ compared to TD children for all measurements, except for the hip external rotation (Table 3), whilst the popliteal angle displayed the greatest differences compared to other joints. These findings support a significant difference in PROM between children with CP and TD children while the effect of walking experience does not have a significant impact on PROM changes.

Good inter-rater reliability was found for most PROM measurements (Table 4). ICC was high (> 0.70 ) for $\mathrm{CP}$ and TD hip abduction, $\mathrm{CP}$ hip external rotation, $\mathrm{CP}$ popliteal angle, and $\mathrm{CP}$ and TD ankle dorsiflexion. ICC was moderate $(0.50-0.70)$ for $\mathrm{CP}$ and TD knee extension, and TD hip external rotation. ICC for the popliteal angle in TD children was 1.00 since $180^{\circ}$ was the default value indicating full knee extension.

\section{Discussion}

This is the first study that recorded the PROM of lower limb joints in young children with CP, GMFCS levels I and II, at the initial stage of walking development. Our findings provide new insights on how the use of an "atypical" gait pattern could potentially influence the changes in PROM. The assessment of PROM of lower limb joints was performed at the onset of the children's independent walking and therefore before any possible PROM restriction due to "atypical" gait patterns. This was repeated at a follow up of 8 months, therefore in a period during which the children practiced walking using an "atypical" gait pattern. Moreover, we compared PROM of children with $\mathrm{CP}$ with those of healthy TD children with similar walking experience in terms of walking duration.

The differences in PROM between the children with $\mathrm{CP}$ and TD children were significant at both the baseline and follow-up measurements, while the PROM changes in time did not differ significantly between the two groups. This observation supports that children with CP have significantly lower PROM at both time points, while walking experience does not significantly impact the PROM neither in children with $\mathrm{CP}$ nor in TD children. Additionally, at baseline, which is a time when all the children were in very early stages of walking, it is unlikely that any decreased values of the PROM were due to particular gait characteristics. This observation strengthens the notion that gait initiation does not contribute to the restriction of PROM in the early stages of walking.

Practically, these observations indicate that the most important factor determining decrease in PROM is not the "normality" of the gait. Therefore, other possible factors should be examined. Absence of selective motor control and low degrees of joint mobility are typical in children with $\mathrm{CP}$ and could have an important impact on PROM; arguing that the less mobile a joint is, the more likely the deformities. More importantly, any kind of possible movement, even if it is not typical, may actually have a positive effect on PROM and muscle elasticity and possibly help avoid PROM restrictions compared to the "lack" of movement; this requires further investigation.

Our study confirms a reduced PROM in children with CP compared to TD children with similar walking experience, specifically in the ankle, hip and knee joints (Table 3, Fig. 1). Similarly, previous studies evaluating PROM in children with CP and TD children reported reduced values of PROM in children with $\mathrm{CP}$, mainly for dorsiflexion, hip abduction and popliteal 


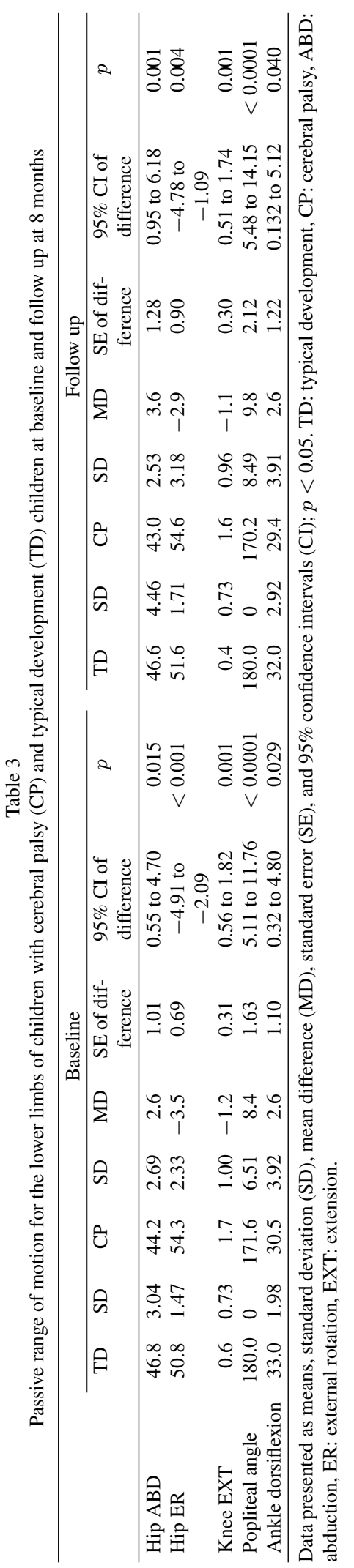


Table 4

Intraclass correlation coefficient (ICC) of passive range of motion between two independent raters

\begin{tabular}{lcccccc}
\hline & \multicolumn{3}{c}{ CP } & & \multicolumn{2}{c}{ TD } \\
\cline { 2 - 3 } \cline { 6 - 6 } \cline { 5 - 6 } & ICC & $95 \%$ CI & & ICC & $95 \%$ CI \\
\hline Hip abduction & 0.74 & 0.46 to 0.87 & & 0.83 & & 0.64 to 0.91 \\
Hip external rotation & 0.92 & 0.75 to 0.97 & & 0.65 & 0.28 to 0.83 \\
Knee extension & 0.64 & 0.26 to 0.83 & & 0.63 & 0.24 to 0.82 \\
Popliteal angle & 0.93 & 0.85 to 0.97 & & 1 & 1 \\
Ankle dorsiflexion & 0.88 & 0.76 to 0.94 & & 0.76 & & 0.51 to 0.88 \\
\hline
\end{tabular}

$\mathrm{CP}$ : cerebral palsy, TD: typical development, CI: confidence intervals.

angle $[6,15,20-22]$. However, the majority of these studies included children older than 4 years and in different stages of their gait development. This fact does not allow us to distinguish whether the recorded differences in PROM could be related to the gait onset, since many of the children were already walking for a long time or they did not walk independently. The only study that included children younger than 4 years was the study by Nordmark et al. [15]. This work suggested that children with CP display a reduced PROM even at younger ages, although it is of interest that the most considerable reductions were recorded after the age of 5 [15]. The aforementioned study is different from ours, as it did not differentiate children based on their ability to walk independently.

In our children with $\mathrm{CP}$ and TD children, decreases in $\mathrm{PROM}$ over time ranged from $0.19^{\circ}$ to $1.38^{\circ}$ and from $0.13^{\circ}$ to $1.06^{\circ}$, respectively. However, there were no significant differences between the two time measurements in either of the groups, showing that walking experience did not have a significant effect on PROM during this time. In addition, the onset of autonomous walking was the most significant change that occurred within the 8-month period and relates directly to our participants' motor development. There were no significant changes in anthropometric characteristics, or frequency and type of treatment, including orthotic devices and medical interventions (Table 1). These findings indicate that the onset of independent gait in children with $\mathrm{CP}$ does not decrease the PROM during the initial stages of independent walking.

At the same time, studies reporting the PROM progression in children with $\mathrm{CP}$, regardless of their age and walking stage, provide a mean annual PROM decrease that depending on the joint varies between $0.75^{\circ}$ and $2.00^{\circ}$ [15]. The same reference values for TD children range between $0.34^{\circ}$ and $1.00^{\circ}$ per year [9]. In our study, the PROM decreases during the first 8 months of independent walking for children with $\mathrm{CP}$ and TD children are in line with these values reported by other researchers during a similar time period $[9,10,15]$. Our observed changes are relatively small but refer to an 8-month period. As age increases these changes could become clinically important in children with $\mathrm{CP}$. The fact that PROM decrease follows a rather expected progression in both $\mathrm{CP}$ and $\mathrm{TD}$, which does not seem to relate to the gait development, supports the notion that gait initiation does not impact the PROM during the first stages of walking.

Children with $\mathrm{CP}$ who cannot walk independently (GMFCS levels III, IV and V) also demonstrate significant restriction in the PROM of lower extremities, which again indicates no direct relationship between gait and PROM restriction. More specifically, Nordmark et al. [15] reported lower range values for hip abduction, popliteal angle and knee extension in children of GMFCS levels III and V, while McDowell et al. [19] reported a significantly decreased PROM in children of lower functional levels. Wright and Bartlett [23] reported increased vertebral deformities in children of GMFCS levels IV and V. Bell et al. [6] showed that the "less functional" children present decreased passive dorsiflexion of the ankle, compared to the "more functional" group.

The observed decreases in the PROM in older children could be attributed to the general tendency of human muscles to lose their elasticity as age progresses. In particular, during normal muscle development, each muscle gradually increases its length in conjunction with the bone on which it is attached. Furthermore, the increase of the diameter of the muscle bundles contributes at least equally to the development of muscle length [24]. As the muscle bundles also develop in thickness, the larger cross sectional area creates greater resistance to elongation. In our study, the children with $\mathrm{CP}$ were 15.5 months older than the TD children due to the fact that children with $\mathrm{CP}$ begin independent walking at an older age. The child's age at the time of PROM measurement could be a factor that influences the final measurement results. Some studies report a strong correlation between age and muscle contracture, suggesting that the PROM decreases as age increases $[6,15,21,25]$. Still, this age difference is a necessary allowance for studies that compare children with $\mathrm{CP}$ and $\mathrm{TD}$ children of similar walking experience.

Indeed, children with CP display dysfunctions regarding their movement and posture from a very early age. The decrease in PROM often appears before the onset of independent walking, or even in children who cannot walk; it also progresses with age. Moreover 
children with lower functional levels and higher GMFCS levels (III-V) seem to demonstrate greater restrictions in PROM compared to children with GMFCS I and II, despite the fact that the latter probably walk using "atypical" gait patterns. Thus, PROM decrease is more likely to relate to lack of movement or muscle growth, than the differentiation of movement due to the use of alternative gait patterns. Our findings support this hypothesis. If this is confirmed in larger studies, therapeutic approaches could encourage active movement and walking based on each child's individual abilities and needs, and irrespective of pattern, at least at a young age.

The period of 8 months between the two measurements and the fact that the measurements were only conducted twice, possibly limits our ability to understand in depth how PROM changes in relation to walking and its duration. However, our choice of this timeframe was based on the clinical reality that children with CP GMFCS levels I and II are subjected to medical interventions at an early age, such as the botulinum toxin injection. Our results also used a small sample size and were based on assessing children with $\mathrm{CP}$ GMFCS levels I and II who can walk independently. The findings encourage further research using larger samples and additional GMFCS levels. Moreover, assessment of musculoskeletal deformities could identify any possible relationship between early PROM changes and deformities in ambulatory children with CP.

This study contributes to expanding our understanding in the effects of independent walking of highly functional children at very young ages. Recording the PROM at the onset of walking excludes probable restriction due to the "atypical" walking patterns of children with CP. It is likely that adopting treatment methods based on any type of movement, and not solely on the normalization of movement, does not deteriorate PROM of the lower limbs. This study suggests that children with $\mathrm{CP}$ could be encouraged to walk using "atypical" gait patterns at least for the first 810 months of their independent walking, without fearing negative influences on their PROM. Further research is required to investigate if this duration can be extended and in what specific ways children could benefit from the independence of this functional activity and treatment. This is of great importance, particularly in an era where emphasis is placed on reinforcing functionality and autonomy of the child, by promoting independent walking as early as possible.

\section{Conclusions}

A significant difference in the PROM between children with CP and TD children was observed at baseline and follow-up measurements. This suggests that the decrease of PROM in children with CP is attributed to other factors, possibly reduced mobility of the specific joints, $\mathrm{CP}$ severity, and age, rather than walking. Compared to baseline, the changes in the passive range of hip abduction and external rotation, knee extension, popliteal angle and dorsiflexion of the ankle in children with $\mathrm{CP}$ were not significantly different after 8 months of independent walking. Similarly, there were no significant changes in the TD children. This provides preliminary data that the alternative gait patterns used by children with $\mathrm{CP}$ do not have a significant impact on PROM during the early stages of walking development. If these findings are confirmed, they can advocate changing the perception of avoiding compensatory movements in fear of PROM changes. In contrast, reinforcing contemporary rehabilitation methods, stressing functionality and participation in clinical practice could be the clinical way forward.

\section{Conflict of interest}

The authors have no conflict of interest to report.

\section{References}

[1] Green LB, Hurvitz EA. Cerebral palsy. Phys Med Rehabil Clin N Am. 2007; 18(4): 859. doi: 10.1016/j.pmr.2007.07. 005 .

[2] Bax M, Goldstein M, Rosembaum P, Leviton A, Paneth N, Dan B, et al. Proposed definition and classification of cerebral palsy. Dev Med Child Neurol. 2005; 47: 571.

[3] Christensen D, Braun K, Doernberg NS, Maenner MJ, Arneson CL, Durkin MS, et al. Prevalence of cerebral palsy, co-occurring autism spectrum disorders, and motor functioning - autism and developmental disabilities monitoring network, USA, 2008. Dev Med Child Neurol. 2014; 56(1): 59. doi: $10.1111 /$ dmcn. 12268 .

[4] Palisano RJ, Hanna SE, Rosenbaum PL, Tieman B. Probability of walking, wheeled mobility, and assisted mobility in children and adolescents with cerebral palsy. Dev Med Child Neurol. 2010; 52(1): 66. doi: 10.1111/j.1469-8749. 2009.03454.x

[5] Ounpuu S, Solomito M, Bell K, DeLuca P, Pierz K. Longterm outcomes after multilevel surgery including rectus femoris, hamstring and gastrocnemius procedures in children with cerebral palsy. Gait Posture. 2015; 42(3): 365. doi: 10 . 1016/j.gaitpost.2015.07.003.

[6] Bell KJ, Ounpuu S, DeLuca PA, Romness MJ. Natural progression of gait in children with cerebral palsy. J Pediatr Orthop. 2002; 22(5): 677. 
[7] Johnson DC, Damiano DL, Abel MF. The evolution of gait in childhood and adolescent cerebral palsy. J Pediatr Orthop. 1997; 17(3): 392.

[8] Narayanan UG. Management of children with ambulatory cerebral palsy: An evidence-based review. J Pediatr Orthop. 2012; 2: S172. doi: 10.1097/BPO.0b013e31825eb2a6.

[9] Mudge AJ, Bau KV, Purcell LN, Wu JC, Axt MW, Selber $\mathrm{P}$, et al. Normative reference values for lower limb joint range, bone torsion, and alignment in children aged 4-16 years. J Pediatr Orthop B. 2014; 23: 15. doi: 10.1097/BPB. Ob013e328364220a.

[10] Soucie JM, Wang C, Forsyth A, Funk S, Denny M, Roach KE et al. Range of motion measurements: Reference values and a database for comparison studies. Haemophilia. 2011; 17: 500. doi: 10.1111/j.1365-2516.2010.02399.x.

[11] Horak FB. Assumptions underlying motor control for neurologic rehabilitation. In Contemporary Management of Motor Control Problems. Proceedings of the II STEP Conference. Fredericksburg VA: Bookcrafters. 1991.

[12] Bobath B, Bobath K. Motor development in the different types of cerebral palsy. London: William Heinmann Medical Books Ltd. 1975.

[13] Ketelaar M, Vermeer A, Hart H, van Petegem-van Beek E, Helders PJ. Effects of a functional therapy program on motor abilities of children with cerebral palsy. Phys Ther. 2001; 81(9): 1534. doi: 10.1093/ptj/81.9.1534.

[14] Bruin M, Smeulders MJ, Kreulen M. Why is joint ROM limited in patients with cerebral palsy? J Hand Surg Eur Vol. 2013; 38(1): 8. doi: 10.1371/journal.pone.0101038.

[15] Nordmark E, Hagglund G, Lauge-Pedersen H, Wagner P, Westbom L. Development of lower limb ROM from early childhood to adolescence in cerebral palsy: A populationbased study. BMC Med. 2009; 7: 65. doi: 10.1186/17417015-7-65.

[16] Rosenbaum PL, Palisano RJ, Bartlett DJ, Galuppi BE, Russell DJ. Development of the gross motor function classification system for cerebral palsy. Dev Med Child Neurol. 2008; 50(4): 249. doi: 10.1111/j.1469-8749.2008.02045.x.
[17] Darrah J, Wiart L, Gorter JW, Law M. Stability of serial range-of-motion measurements of the lower extremities in children with cerebral palsy: Can we do better? Phys Ther 2014; 94(7): 987. doi: 10.2522/ptj.20130378.

[18] Kilgour GM, McNair PJ, Stott NS. Intrarater reliability of lower limb sagittal range-of-motion measures in children with spastic diplegia. Dev Med Child Neurol. 2003; 45(6): 391.

[19] McDowell BC, Hewitt V, Nurse A, Weston T, Baker R. The variability of goniometric measurements in ambulatory children with spastic cerebral palsy. Gait Posture. 2000; 12(2): 114.

[20] McDowell BC, Salazar-Torres JJ, Kerr C, Cosgrove AP. Passive range of motion in a population-based sample of children with spastic cerebral palsy who walk. Phys Occup Ther Pediatr. 2012; 32(2): 139. doi: 10.3109/01942638.2011.644032.

[21] Barber L, Hastings-Ison T, Baker R, Barrett R, Lichtwark G. Medial gastrocnemius muscle volume and fascicle length in children aged 2 to 5 years with cerebral palsy. Dev Med Child Neurol. 2011; 53(6): 543. doi: 10.1111/j.1469-8749. 2011.03913.X

[22] Kilgour GM, McNair PJ, Stott NS. ROM in children with spastic diplegia, GMFCS i-II compared to age and gender matched controls. Phys Occup Ther Pediatr. 2005; 25(3): 61.

[23] Wright M, Bartlett DJ. Distribution of contractures and spinal malalignments in adolescents with cerebral palsy: Observations and influences of function, gender and age. Dev Neurorehabil. 2010; 13(1): 46. doi: 10.3109/1751842090326 7101.

[24] Benard MR, Harlaar J, Becher JG, Huijing PA, Jaspers RT. Effects of growth on geometry of gastrocnemius muscle in children: A three-dimensional ultrasound analysis. Journal of Anatomy. 2011; 219(3): 388. doi: 10.1111/j.1469-7580.2011. 01402.x.

[25] Svehlik M, Leistritz L, Kraus T, Zwick EB, Steinwender G, Linhart WE. The growth and the development of gastro-soleus contracture in cerebral palsy. Gait Posture. 2013; 38: S1. doi: 10.1016/j.gaitpost.2013.07.032. 